\title{
Elaboration d'un outil dynamique d'évaluation des dommages de crues sur l'ensemble de la région Ile-de-France
}

\section{A dynamic tool to estimate flood damages in the Ile-de-France administrative region}

\author{
par L. Gazull \\ Stratégis SA
}

Avec la participation de : l'agence de l'eau Seine Normandie, les Grands Lacs de Seine, la région Ile de France, le ministère de l'Environnement.

In 1990, a large study program has been initiated by "Les grands lacs de Seine " Institute to estimate the impact of existing dams and to calculate the profitability rate of new projects.

The content of this new stage of study is the design and the development of a decision making software to reduce flood damages in all the lle de France administrative region.

This software helps to define a planning scenario, to launch an hydraulic simulation of flood, to calculate flood damages and to analyse the economic impact. Designed around a Geographic Information System (GIS), it can produce maps to analyse results at different scales from communal contours to region area.

A detailed relational database has been designed to manage the huge mass of information collected along the $500 \mathrm{~km}$ of river and the 300 communes concerned.

This tool represents not only a simulation code but also a priceless information source on historic floods, local protections and vulnerable equipments.

\section{I — OBJECTIFS DE L'OUTIL}

L'objectif principal de l'outil mis en place est d'estimer l'impact en termes économiques des ouvrages de lutte contre les inondations existants ou futurs, à l'échelle de la région Ile de France.

A cet effet, l'outil doit intégrer l'ensemble des fonctionnalités permettant :

- de définir une hydrologie influencée ou non par les barrages,

- de choisir un certain nombre d'aménagements du lit mineur et du champ majeur,

- de simuler leur devenir hydraulique à travers les lits (mineurs et majeurs) de la Seine, la Marne et l'Oise,

- de calculer les dommages directs et indirects afférants aux inondations,

- d'analyser les résultats et impacts aux échelles adaptées à la problématique.

Le présent article vise à décrire les choix effectués et la méthodologie empruntée pour développer cet outil.

\section{II $\square$ CONTRAINTES MÉTHODOLOGIQUES}

Les nombreux objectifs de l'étude se traduisent pas un grand nombre de contraintes ayant orienté les choix tant en matière de modélisation, structuration et représentation des données qu'en matière d'outils utilisés.

\subsection{Une étude à caractère régional :}

Le linéaire total de rivière à traiter atteint près de $500 \mathrm{~km} ; 300$ communes sont concernées par les inondations de la Seine, la Marne et l'Oise dans la traversée de l'Ile de France ; 2500 casiers hydrauliques ont été créés pour représenter le lit majeur.

La caractérisation de l'occupation des sols dans toute la zone inondable, le recensement des équipements vulnérables et l'estimation des enjeux associés représentent des masses d'information considérables.

De plus, ces informations sont évolutives :

- L'IAURIF (Institut d'Aménagement et d'Urbanisme de la Région Ile de France) met à jour le MOS (Mode d'Occupation des Sols) de la région Ile de France tous les 4 ans. 
- L'INSEE prévoit un nouveau recensement général de la population en 1998.

Enfin, l'information doit pouvoir être restituée à différents niveaux géographiques ou administratifs en fonction de la pertinence des paramètres étudiés.

Le moteur de base de données choisi doit donc être à même d'accepter de nombreuses bases volumineuses et proposer un modèle suffisamment évolué pour gérer les interrelations qui existent entre toutes ces données.

\subsection{Un outil opérationnel à remettre au maître d'ouvrage}

La seconde contrainte est que l'outil développé doit non seulement permettre aux bureaux d'étude de produire les résultats escomptés permettant d'estimer la rentabilité des aménagements projetés mais il doit également être livré au maître d'ouvrage afin qu'il puisse simuler à son gré un certain nombre d'aménagements.

L'outil doit donc être " robuste ", simple d'emploi et pouvoir être installé à moindre coût dans une structure ne disposant pas de moyens informatiques lourds.

\subsection{Un nombre " illimité " de scénarios hydrauliques à évaluer}

Un scénario de simulation se définit comme une simulation de calculs hydrauliques et socio-économiques correspondant à une crue donnée dans un contexte d'aménagements hydrauliques et d'occupation des sols.

L'utilisateur final peut modifier un grand nombre de paramètres hydrologiques et hydrauliques préétablis et ainsi redéfinir les caractéristiques de l'écoulement dans chaque casier.

La structure des données doit donc être suffisamment souple pour pouvoir créer et accéder aux résultats d'une simulation en un temps acceptable pour l'utilisateur.

\subsection{Une grande diversité " d'objets géographiques" à manipuler}

L'estimation des dommages directs et indirects nécessite la manipulation d'un grand nombre d'objets géographiques très divers :

- Objets ponctuels : transformateurs EDF, centraux téléphoniques, ...

- Objets surfaciques : zones du MOS, îlots INSEE, enceintes spécifiques ...

- Objets linéaires : réseau routier,

- Objets RASTER : Images, photographies aériennes, scannérisation IGN SCAN25.

Bien que tous les objets ne soient pas présents dans l'outil final pour raison de confidentialité, ils participeront tous à l'estimation des dommages directs et indirects.

La première phase de la présente étude a prouvé l'intérêt indiscutable de l'utilisation d'un SIG dans ce type de problématique. Le SIG intégré à l'outil doit pouvoir gérer l'ensemble des objets décrits ci-dessus et être suffisamment " ouvert " pour accepter des liens avec les données externes issues de simulations.

\subsection{Une grande variété de résultats à produire}

Enfin, la dernière contrainte est que la valorisation des nombreux résultats produits par l'étude passe par une mise en forme adaptée aux différents indicateurs calculés et aux objectifs visés.
Cette mise en forme comprend :

- des graphiques simples permettant de comparer visuellement les résultats entre les différentes entités ou entre deux scénarios de simulation,

— des représentations cartographiques à différentes échelles adaptées à la précision des résultats,

- des tableaux clairs résumant les caractéristiques hydrauliques et socio-économiques pour chaque échelle de traitement...

\section{III — STRUCTURE DE L'OUTIL}

Au vu de l'ensemble des objectifs des différents maîtres d'ouvrage, l'outil développé peut se définir comme suit :

- un outil de simulation hydraulique et socio-économique des crues à l'échelle régionale ;

- un outil cartographique d'information sur les crues historiques ;

- un outil de suivi des aménagements (protections linéaires, singularités hydrauliques) ;

- un outil d'analyse des impacts économiques des aménagements hydrauliques existants ou futurs.

Les expériences en cours ou passées (SCARAUZI, MIKEGIS, CALIPSEAU) ont montré que les outils SIG pouvaient représenter d'excellents supports de valorisation des modèles hydrauliques. Elles ont également montré les limites de ces outils notamment dans la structuration des données, la prise en compte des séries temporelles, la rapidité d'exécution de certains traitements, etc.

La tendance actuelle est d'utiliser le SIG comme un simple maillon d'une chaîne de traitement faisant appel à des outils de développement puissants, des SGBDR externes, des bibliothèques statistiques et graphiques.

\subsection{Environnement matériel et logiciel}

L'environnement nécessaire pour le fonctionnement de l'outil comprend :

- un micro-ordinateur type PENTIUM 133 équipé de WINDOWS 95 ou WINDOWS NT 4.0 ;

- une imprimante couleur A4/A3 de type jet d'encre ;

- un écran haute résolution 17 pouces ;

- le logiciel SIG MAPINFO V4.x.

Cette configuration est donc tout à fait " classique " dans le contexte informatique actuel.

DELPHI 3.0 a été choisi comme environnement de développement. Les bases de données sont directement structurées et pilotées par le moteur BDE de DELPHI.

Cet environnement de développement dispose de l'ensemble des bibliothèques graphiques et des utilitaires d'édition nécessaires à la mise en forme des productions de l'étude.

Une interface spécifique a été développée afin de permettre à un utilisateur ne connaissant pas MAPINFO de produire et analyser aisément ses résultats. Pour ce faire, les fenêtres MAPINFO sont pilotées à travers des liens OLE directement à partir de l'interface DELPHI.

\subsection{Choix d'un fond de plan de référence}

Le choix d'un fond de plan cartographique de référence est primordial pour la sortie des résultats et pour le repérage des informations. 
Au MOS de l'IAURIF, il a été préféré le produit IGN SCAN25 dont dispose la DIREN Ile de France.

Ce fond est une " image " géoréférencée des cartes IGN au 1/25000. Ce fond en niveau de gris ou en noir et blanc permet de se repérer facilement sans pour autant surcharger l'information graphique.

\subsection{Données géographiques de base et modèle de données \\ - Les casiers hydrauliques définis par Hydratec}

Zone élémentaire de stockage de l'eau dans le champ majeur.

Le contour du casier est délimité suivant la nature des obstacles, la topographie locale, le Mode d'Occupation des Sols. La topographie d'un casier est représentée par une courbe donnant le pourcentage de surface inondée en fonction de la cote.

\section{- Les stations hydrométriques}

Repère ponctuel sur le réseau hydrographique au droit duquel ont été observés des niveaux de crue historiques. En ce même repère les hydrogrammes et limnigrammes des crues simulées pourront également être consultés.

\section{- Le MOS}

Le MOS est un document numérique établi au $1 / 5000^{\circ}$ par l'IAURIF. Il représente une carte d'occupation du sol classifié en 110 postes. Le MOS a été enrichi par croisement géographique et statistique avec les îlots INSEE afin d'estimer dans chaque zone du MOS, le nombre de logements et le nombre d'habitants y figurant.
- Les îlots INSEE et les estimations d'emplois par îlots (IAURIF)

Ces deux bases de données permettent de connaître dans chacun des îlots de la petite couronne le nombre de salariés répartis dans les 14 classes de la nomenclature NAP15.

\section{- Les équipements sensibles}

Ces bases rassemblent un grand nombre d'équipements ponctuels dont la mise hors service a des conséquences graves sur le fonctionnement général de l'activité en région Ile de France.

Ces informations sont issues de l'IAURIF, d'EDF-GDF, de la SNCF, de la RATP...

\section{- Le réseau routier principal}

Cette couche d'information est fournie par l'IAURIF. Elle est enrichie à partir de la topographie du terrain naturel pour renseigner les points bas de chaque tronçon routier. Par croisement avec la zone inondable elle permet d'estimer les perturbations de trafic.

\section{- Les spécificités communales}

Cette base comprend un ensemble de paramètres permettant de caractériser l'occupation des sols et les enjeux socio-économiques au sein de chaque commune. Elle est renseignée par repérage de terrain et à partir de données INSEE.

La plupart des informations sont ramenées au niveau du casier hydraulique qui représente l'unité spatiale d'intégration fondamentale. Le modèle conceptuel des données figurant page suivante présente un aperçu de la complexité des relations entre objets manipulés.

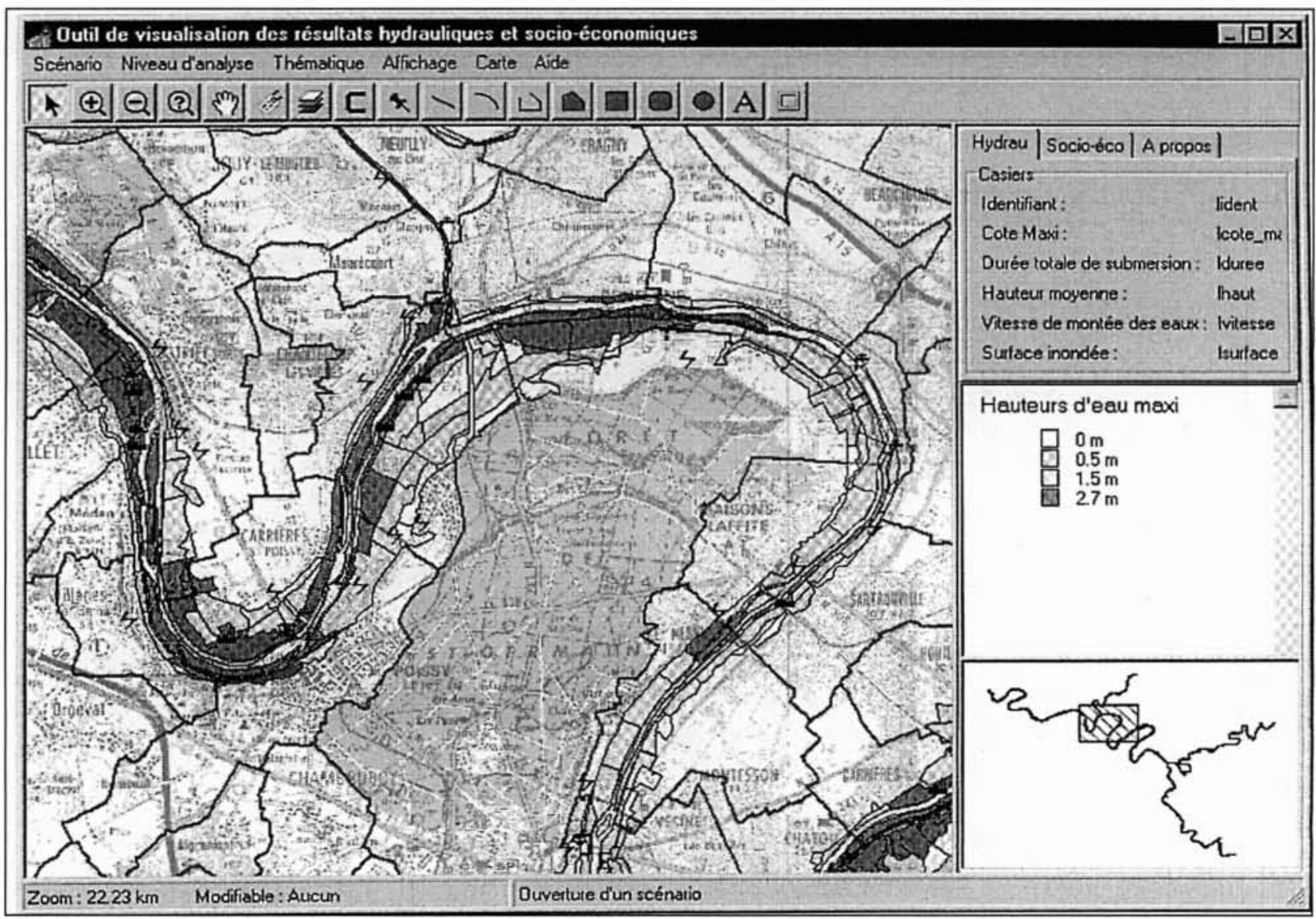

1. Exemple d'interface du module 4 


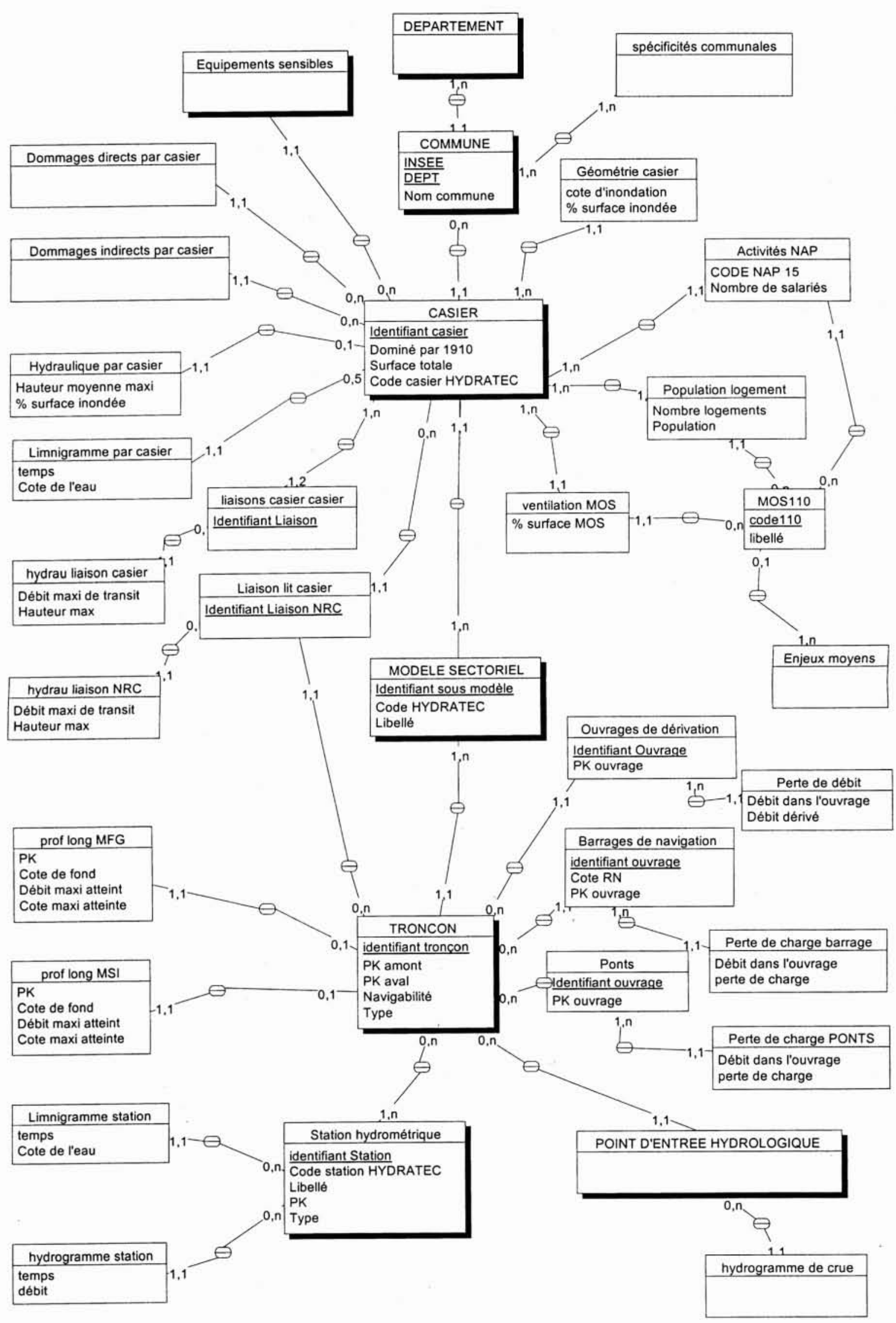

2. Modèle conceptuel général des données. 


\subsection{Une structure modulaire}

L'outil d'évaluation s'articule autour de 6 modules :

1. Un module de gestion des scénarios de simulation,

2. Un module de simulation hydraulique (développé par la société Hydratec),

3. Un module de simulation socio-économique,

4. Un module de visualisation des résultats hydrauliques et socio-économiques,

5. Un module de mise à jour des données de base,

6. Un module d'analyse spatiale des résultats de simulation.

\section{IV — MÉTHODOLOGIE DE CALCUL}

Une session classique de travail sur l'outil peut se décomposer en quatre temps :

1. la définition d'un scénario,

2. les calculs hydrauliques,

3. les calculs socio-économiques,

4. l'analyse des résultats.

\section{- 4.1 La définition d'un scénario}

La définition d'un scénario est l'étape préalable à toute nouvelle simulation hydraulique ou socio-économique.

\subsection{Les calculs hydrauliques}

La méthodologie retenue pour la modélisation hydraulique n'est pas décrite dans le présent article. Elle fait l'objet d'une publication à part présentée par la société HYDRATEC.

\section{- 4.3 Les calculs socio-économiques}

Les dommages directs sont calculés pour deux types d'entités géographiques :

- Les casiers hydrauliques: L'ensemble des données socioéconomiques précédemment citées sont ramenées soit directement soit statistiquement à la surface du casier.

La répartition surfacique du MOS est obtenue par croisement géographique entre les zones MOS et les contours des casiers. Les logements et la population sont répartis par casier et par type de MOS par croisement géographique et traitement statistique entre les zones MOS, les contours des casiers et les contours des îlots INSEE.

Les emplois sont répartis par casier, par type de MOS et par classe NAP15, par croisement géographique et traitement statistique entre les zones MOS, les contours des casiers et les contours des îlots INSEE.

Les données communales (INSEE ou autres) sont appliquées sur tous les casiers contenus dans la commune.

L'aléa hydraulique (hauteur moyenne et durée moyenne de submersion) est considéré uniforme sur toute la surface du casier.

- Les équipements sensibles: Les données précédemment citées sont fonction du type d'équipement et des renseignements collectés.
L'aléa hydraulique (hauteur et durée de submersion) est considéré au droit de l'équipement.

La hauteur d'eau est calculée précisément si l'on connât la cote plancher de l'équipement ou si elle est considérée égale à celle obtenue dans le casier contenant l'équipement.

La durée de submersion est considérée égale à celle obtenue dans le casier contenant l'équipement.

Les dommages indirects affectant les réseaux de distribution et de transport sont estimés globalement à l'échelle de la région ou d'une vallée à partir d'indicateurs locaux simples (hauteur d'eau au pont d'Austerlitz, points bas des principaux axes routiers et ferroviaires ...). La relation entre ces indicateurs et l'endommagement est établie préalablement en concertation avec les gestionnaires des réseaux après étude de scénarios types.

\subsection{L'analyse des résultats}

L'analyse des résultats se fait à travers différents modes de représentation et différents outils mis à disposition de l'utilisateur. L'analyse peut se faire scénario par scénario ou par comparaison de deux scénarios entre eux.

Trois types d'outils sont proposés :

- un outil tableur présentant les résultats sous forme de tableaux ;

- un outil grapheur présentant les résultats sous forme de graphiques (barres et camemberts) ;

- un outil cartographique présentant les résultats sous forme de cartes en plages colorées ou de symboles.

Pour chaque type d'outils les résultats peuvent être analysés au niveau des équipements sensibles, des casiers, des communes, des départements et de la région.

Cependant la précision de certains paramètres ne permettra une analyse pertinente qu'au niveau d'un ensemble de communes, voire de l'ensemble de la région.

Tous les résultats sont imprimables directement au format A4 ou A3.

\section{$V \square$ CONCLUSION}

L'outil mis en place a été conçu pour répondre à une problématique à l'échelle de la région. Son échelle de travail la mieux adaptée est de l'ordre du $1 / 25000^{\circ}$.

Les objectifs ambitieux ont parfois contraint à simplifier les unités de travail, voire limiter les fonctionnalités de l'outil au profit de sa souplesse d'utilisation, d'une plus grande rapidité d'exécution et dans le souci d'une meilleure pertinence des résultats.

Cet outil est l'aboutissement d'un travail très important engagé en 1992 en matière de recueil de données et d'investigations méthodologiques, tant de la part des chargés d'étude que des maîtres d'ouvrage.

A cet égard il constitue non seulement un code de simulation mais également une base de connaissances actualisables sur les crues historiques, les protections existantes et les équipements sensibles en zone inondable. 